This item was submitted to Loughborough's Research Repository by the author.

Items in Figshare are protected by copyright, with all rights reserved, unless otherwise indicated.

\title{
Performance analysis of hybrid UAV networks for probabilistic content caching
}

PLEASE CITE THE PUBLISHED VERSION

https://doi.org/10.1109/JSYST.2020.3013786

PUBLISHER

IEEE

VERSION

AM (Accepted Manuscript)

\section{PUBLISHER STATEMENT}

Personal use of this material is permitted. Permission from IEEE must be obtained for all other uses, in any current or future media, including reprinting/republishing this material for advertising or promotional purposes, creating new collective works, for resale or redistribution to servers or lists, or reuse of any copyrighted component of this work in other works.

\section{LICENCE}

\section{All Rights Reserved}

\section{REPOSITORY RECORD}

Khuwaja, Aziz Altaf, Yongxu Zhu, Gan Zheng, Yunfei Chen, and Wei Liu. 2021. "Performance Analysis of Hybrid UAV Networks for Probabilistic Content Caching”. Loughborough University.

https://hdl.handle.net/2134/17000362.v1. 


\title{
Performance Analysis of Hybrid UAV Networks for Probabilistic Content Caching
}

\author{
Aziz Altaf Khuwaja, Yongxu Zhu, Member, IEEE, Gan Zheng, Senior Member, IEEE, Yunfei Chen, Senior \\ Member, IEEE, and Wei Liu, Senior Member, IEEE
}

\begin{abstract}
Caching content in small-cell networks can reduce the traffic congestion in backhaul. In this paper, we develop a hybrid caching network comprising of unmanned aerial vehicles (UAVs) and ground small-cell base stations (SBSs), where UAVs are preferred because of their flexibility and elevated platform for line-of-sight. First, we derive the association probability for the ground user affiliated with a UAV and ground SBS. Then, we derive the successful content delivery probability by considering both the inter-cell and intra-cell interference. We also analyze the energy efficiency of the hybrid network and compare it with the separate UAV and ground networks. We further propose the caching scheme to improve the successful content delivery by managing the content popularity, where the part of the caching capacity in each UAV and ground SBS is reserved to store the most popular content (MPC), while the remaining stores less popular contents. Numerical results unveil that the proposed caching scheme has an improvement of $26.6 \%$ in content delivery performance over the MPC caching which overlooks the impact of content diversity during caching.
\end{abstract}

Index Terms-Caching, content delivery performance, energy efficiency, unmanned aerial vehicle (UAV)

\section{INTRODUCTION}

Wireless edge caching is one of the most promising solutions to backhaul congestion. In the wireless local caching, the popular contents are stored in caches at the network edges, such as small cells and hand-held devices during offpeak time [1], [2]. Consequently, contents can be directly requested and accessed locally by users during peak times to reduce the burden on backhaul. In addition to latency, the energy efficiency of such networks is also important from the network planning viewpoint [3], as the energy efficiency performance is compelling for understanding the impact of network parameters, such as BSs density, power consumption,

This work is supported in part by EC H2020 DAWN4IoE-Data Aware Wireless Network for Internet-of-Everything (778305) and in part by the Royal Society's International Exchanges Scheme under the grant number IEC $\backslash$ NSFC $\backslash 181395$ and in part by UK EPSRC under grant number EP/N007840/1, and in part by the Leverhulme Trust Research Project Grant under grant number RPG-2017-129.

A. A. Khuwaja is with the School of Engineering, University of Warwick, Coventry, U.K. CV4 7AL and also with the Department of Electrical Engineering, Sukkur IBA University, Sukkur 65200, Sindh, Pakistan (e-mail: A.khuwaja@warwick.ac.uk).

Y. Zhu is with the Division of Computer Science and Informatics, London South Bank University, London, SE1 0AA, U.K. (e-mail: zhuy6@1sbu.ac.uk).

G. Zheng is with the Wolfson School of Mechanical, Electrical, and Manufacturing Engineering, Loughborough University, Leicestershire, LE11 3TU, U.K. (e-mail: g.zheng@ @lboro.ac.uk).

Y. Chen is with the School of Engineering, University of Warwick, Coventry, U.K. CV4 7AL (e-mail: Yunfei.Chen@warwick.ac.uk).

W. Liu is with the the State Key Laboratory of Integrated Services Networks, Xidian University, China. (e-mail: liuweixd@mail.xidian.edu.cn). and the quality-of-service requirement. Thus, it is of great interest to study the energy efficiency performance of a hybrid caching network to successfully deliver the cached contents to the ground user for the required threshold and then compare it with the separate unmanned aerial vehicle (UAV) and ground networks.

UAV can provide flexible access due to their maneuverability and hence, can be exploited as an aerial BS or a cache to facilitate the high-speed transmission [4]. Different from the network caching using the fixed ground infrastructure, the UAV-enabled caching can increase the likelihood of successful content transmission because of their flexibility which can take the caching content closer to the typical user. However, the deployment of UAVs can encounter many challenges [5], and it can be based either on the deterministic approach, such as circle packing method in [6] and hexagonal placement in [7], or on the random distribution using tools of stochastic geometry.

Recently, the research on the coexistence of the UAVenabled network with the terrestrial network was presented in [8]- [11]. For these works, the spatial distribution of base stations is defined by a Poisson point process (PPP). For instance, in [8]- [11], UAVs were distributed according to 3D PPP and the terrestrial network was modeled by PPP. However, most of these studies ignore the caching aspect in UAVs. In [12], the authors provided an analytical framework using independent PPPs for UAV and ground BSs to evaluate the performance of UAV assisted cellular network in terms of signal-to-interference-plus-noise ratio (SINR) coverage probability. In [13], the authors modeled a multi-layer aerial network with PPP using air-to-ground (AG) and air-to-air (AA) channel models to evaluate the transmission probability and area spectral efficiency. In [14], the probabilistic caching placement was investigated in heterogeneous UAV network modeled as an independent homogeneous PPP without considering cochannel interference or the terrestrial network. In [15], the authors presented a framework for the uplink transmission of cached contents from the ground SBSs distributed according to a homogeneous PPP to a single aerial user equipment over the ground-to-air (GA) channel. In [16], the UAV-assisted secure transmission was studied via caching, where UAVs offload the video traffic and deliver to mobile users in small cells. On the other hand, the authors in [17] presented the architecture of caching in UAV-enabled small-cell network. However, an analytical framework for the performance analysis has not been provided. The works in [18]- [20] utilized the cacheenabled UAVs in radio access networks. However, these works 
do not model their system as a multi-tier caching network that consists of ground SBSs to supplement UAVs.

Content placement is a key challenge due to the limited caching capacity in SBSs, because spontaneous caching in nearby SBSs will incur more interference. Extensive research has been conducted to analyze the joint content placement and transmission performance as well as designing relevant caching strategies. For instance, [21] studied the optimal content placement of the cache-enabled heterogeneous cellular network. [22] proposed a caching strategy for the clustercentric small-cell network that combines the most popular content (MPC) and the large content diversity. This strategy was extended in [23] to the distributed relay network to improve outage performance. In [24], heuristic solution was proposed to significantly improve the video delivery performance in the cache-enabled wireless heterogeneous network. In [25], the authors proposed a heuristic algorithm to maximize the transmission performance of cache-enabled multi-antenna and millimetre-wave small-cell networks. [26] analyzed and optimized the performance of the content placement in the terrestrial BSs and in mobile users that coexists in a heterogeneous wireless network. In [27], the cache-enabled nodes were grouped in disjoint clusters by Matern hard core point process. In [28], spatial caching strategy was proposed to improve content delivery probability and to avoid caching redundancy in a heterogeneous network. In [29], the authors studied the techniques to enhance the caching capacity in the mobile ad hoc networks. However, these works did not consider the content placement scheme in UAV-enabled networks using the realistic AG channel conditions in urban environments.

Small-cell networks are expected to provide significant improvement in the content delivery for higher transmission rates and reduced backhaul congestion. In the previous works [21]- [29], cache-enabled networks were designed mostly for the ground BSs with fixed infrastructure. However, their installation can be hampered by geographical constraints in different urban environments. On the other hand, UAVs provide flexible platforms to deploy as aerial BSs or aerial caches in urban settings. However, the coexistence of the aerial and terrestrial SBSs in a multi-tier caching network has been largely ignored in [18]- [29].

The existing studies mentioned above have neither considered the modeling of a hybrid caching network consisting of UAVs and ground SBSs randomly located according to the PPP [30], [31], nor analyzed its content delivery and energy efficiency performance. Therefore, in this paper, we present the guidelines to model the hybrid caching network and analyze the performance of the proposed content caching scheme which exploits the content diversity based on its popularity measures. However, the use of protocol architecture of a specific 3rd generation partnership project (3GPP) standard in a hybrid caching network is beyond the scope of this paper. The main contributions of this work are summarized as follows:

1) User association probability for the UAVs and the ground SBSs are derived using stochastic geometry. Both UAVs and ground SBSs coexist in a network where we adopt the density sharing scheme for the adequate deployment of UAVs and ground SBSs. Their locations are determined by the homogeneous PPPs. The typical user request for a particular file is highly likely to be associated with the cache-enabled UAV and ground SBS in a hybrid network based on the maximum received power criteria.

2) Successful content delivery probability is used to analyze the network performance. Furthermore, inter-tier and intra-tier interferences are taken into account and modeled by the Laplace transforms. The results reveal that the successful content delivery performance is dependent on the network parameters, such as the UAV and SBS density to control the interference and the UAV altitude, and also on the content-related parameters, such as the size of the content in the database, caching capacity of UAVs and SBSs, skewness in content popularity, and the target data rate.

3) Energy efficiency is a performance metric defined as the ratio of the area spectral efficiency for the successful content delivery to the average power consumption of the network for a given quality-of-service threshold [32]. The energy efficiency of the cache-enabled hybrid network is then compared with those of the separate UAV and ground networks to assess the effectiveness of the hybrid approach.

4) A hybrid caching scheme is proposed in which a portion of the caching capacity in each UAV and SBS is designated to cache the MPC with the higher popularity probability. Thereafter, the contents with the moderate popularity are stored in the remaining portion of the caching capacity. Furthermore, the content delivery performance of the proposed caching scheme is compared with the widely used popular content placement method in [22]- [25] as a baseline scheme which only cache MPC.

\section{SYSTEM MODEL}

In this section, we will describe the network topology, the channel model, and the content placement scheme for the cache-enabled hybrid network illustrated in Fig. 1. The commonly used symbols in this paper and their meanings are tabulated in Table I.

\section{A. Network Topology}

We consider a three-tier time division multiplexing heterogeneous network with UAVs in tier U, ground SBSs in tier $\mathrm{G}$, and one macro BS for backhaul connectivity. Specifically, UAVs are deployed as a cache-enabled aerial BSs in tier U after the ground SBSs are deployed, where UAVs share the same altitude $h$ and transmit with power $P_{\mathrm{U}}$. In this case, the random deployment of UAVs follow the PPP in [30], [31] due to two main reasons. First, the projection distribution is similar to the classic PPP when all UAVs hovers at the same altitude. Second, this offer analytical tractability for modeling the uncertainty in the deployment of the cache-enabled UAVs in the worst-case scenario [8]- [11]. In tier G, SBSs are on the ground and transmit with power $P_{\mathrm{G}}$. Furthermore, we 
TABLE I

SUMMARY OF SYMBOLS.

\begin{tabular}{c|l}
\hline Symbols & Meaning \\
\hline$\Phi_{\mathrm{U}}, \Phi_{\mathrm{G}}$ & Location of UAVs in tier U, SBSs in tier G \\
\hline$\lambda_{\mathrm{U}}, \lambda_{\mathrm{G}}$ & Density of UAVs in tier U, SBSs in tier G \\
\hline$h$ & UAV altitude \\
\hline$\eta$ & Density control factor \\
\hline$K$ & Size of database \\
\hline$J$ & Caching capacity of each UAV and SBS \\
\hline $\mathrm{z}$ & $\begin{array}{l}\text { State of UAV being in line-of-sight (z }=\mathrm{L}) \text { and non } \\
\text { line-of-sight }(\mathrm{z}=\mathrm{N}) \text { conditions }\end{array}$ \\
\hline$f_{k}$ & The $k$-th file \\
\hline$b_{k}$ & $\begin{array}{l}\text { Placement probability of file } f_{k} \text { in UAV and SBS } \\
\text { cache }\end{array}$ \\
\hline$m_{k}$ & Popularity measure for the requested file $f_{k}$ \\
\hline$g_{o}$ & Rayleigh fading gain with unit mean for desired link \\
\hline$g_{i}, g_{j}, g_{l}$ & $\begin{array}{l}\text { Rayleigh fading gain with unit mean for interference } \\
\text { link }\end{array}$ \\
\hline
\end{tabular}

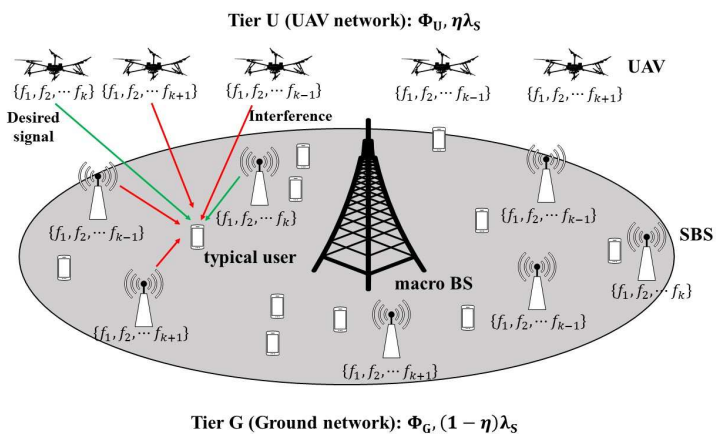

Fig. 1. System model for cache-enabled hybrid network with UAVs and ground SBSs deployed according to PPP.

assume that each UAV and SBS is equipped with a single antenna. In this paper, we consider a density sharing scheme for appropriate participation of the cache-enabled UAVs and ground SBSs in a hybrid network. The overall distribution of the cache-enabled nodes in a hybrid network is modeled by a homogeneous PPP $\Phi_{\mathrm{S}}$ with density $\lambda_{\mathrm{S}}$. The UAVs and the ground SBSs in tier $U$ and tier $G$ follows two independent homogeneous PPPs $\Phi_{\mathrm{U}}$ and $\Phi_{\mathrm{G}}$ with densities $\lambda_{\mathrm{U}}=\eta \lambda_{\mathrm{S}}$ and $\lambda_{\mathrm{G}}=(1-\eta) \lambda_{\mathrm{S}}$, respectively. In this case, $0 \leq \eta \leq 1$ and the factor $1-\eta$ defines the percentage of active SBSs in a hybrid network with $\lambda_{\mathrm{S}} \geq \lambda_{\mathrm{U}}$ and $\lambda_{\mathrm{S}} \geq \lambda_{\mathrm{G}}$. Also, the ground users are spatially distributed according to an independent homogeneous PPP $\Phi_{\mathrm{T}}$ with density $\lambda_{\mathrm{T}}$. We assume that the user density is much larger than the UAVs and SBSs densities $\left(\lambda_{\mathrm{T}} \gg \lambda_{\mathrm{U}}\right.$ and $\left.\lambda_{\mathrm{T}} \gg \lambda_{\mathrm{G}}\right)$. From the Slivnyak's theorem of PPP in [30], the user association probability for every pair of UAV-user is the same. Thus, the typical user is set as a ground reference point which is served by each UAV and SBS at each time slot with multiple active users at each time slot in macro BS.

\section{B. Channel Model}

For the downlink communication in tier $\mathrm{U}$, we have an AG channel between UAV and the typical user. In such a channel, the path-loss is dependent on the environment and the propagation distance. In this case, the line-of-sight (LOS) and non line-of-sight (NLOS) links can be considered as separate components of the AG channel. Thus, the path-loss for LOS and NLOS links are given as [33]

$$
L_{\mathrm{U}, \mathrm{z}}(X)=\beta_{\mathrm{o}} X^{\alpha_{\mathrm{z}}} ; \quad \mathrm{z} \in\{\mathrm{L}, \mathrm{N}\},
$$

where $\beta_{\mathrm{o}}=\left(\frac{4 \pi f}{c}\right)^{2}$ is the frequency-dependent channel power at the reference distance of $1 \mathrm{~m}, f$ is the carrier frequency, $c$ is the speed of light, $z \in\{L, N\}$ denotes the condition of being $\operatorname{LOS}(\mathrm{z}=\mathrm{L})$ or NLOS $(\mathrm{z}=\mathrm{N})$ links, $X=\sqrt{d^{2}+h^{2}}$ is the propagation distance, $d$ is the user distance from the projection of the UAV, $\alpha_{\mathrm{L}}$ and $\alpha_{\mathrm{N}}$ are the path-loss exponents for the LOS and NLOS links, respectively. Also, the probability of having a LOS link is given as [34]

$$
p_{\mathrm{L}}(X)=\frac{1}{1+a \exp \left(-b\left[\frac{180}{\pi} \sin ^{-1}\left(\frac{h}{X}\right)-a\right]\right)},
$$

and the probability of having a NLOS link is given by $p_{\mathrm{N}}(X)=1-p_{\mathrm{L}}(X)$, where $a$ and $b$ are constants related to the environment. In order to have tractable analysis we consider that the LOS probability for different communication links are independent [11]

In the ground network, the channel between SBS and the typical user has path-loss given as

$$
L_{\mathrm{G}}(Y)=\beta_{\mathrm{o}} Y^{\alpha_{\mathrm{G}}}
$$

where $Y$ is the user distance from the ground SBS to typical user and $\alpha_{\mathrm{G}}$ is the path-loss exponent of tier G.

\section{Probabilistic Content Placement Scheme}

We assume that the particular contents (such as multimedia files) are placed in the cache at both the UAVs and ground SBSs. A typical user randomly requests the contents from the finite content database $\mathcal{C}:=\left\{f_{1}, \cdots, f_{k}, \cdots, f_{K}\right\}$, where the database size is $K$ and the $k$-th file is requested with a probability of $m_{k}$. We assume that the contents differ in popularity for all $k$ and the files are requested in decreasing popularity, so that $m_{k}>m_{k+1}>\cdots>m_{K}$ and $\sum_{k=1}^{K} m_{k}=1$. We consider that all content files have the same normalized size equal to 1 [35]. Furthermore, we assume that each SBS can only store up to $J$ contents, where $J \leqslant K$. Such an assumption is practical because not all SBSs have enough capacity to store the entire database contents.

In this paper, we adopt the probabilistic content placement scheme used in [14], [21], and [25] for caching at UAVs and ground SBSs while considering their storage capacity. In this scheme, the caching probability $b_{k}$ satisfies the following condition

$$
\sum_{k=1}^{K} b_{k} \leq J, \quad 0 \leq b_{k} \leq 1, \forall k .
$$

The condition in (4) allow each UAV and SBS to cache the maximum amount of the total content up to their caching capacity $J$. Note that, using the probabilistic content placement strategy, $\Phi_{\mathrm{G}, k}$ and $\Phi_{\mathrm{U}, k}$ are independent PPPs with densities $\lambda_{\mathrm{G}, k}=b_{k} \lambda_{\mathrm{G}}$ and $\lambda_{\mathrm{U}, k}=b_{k} \lambda_{\mathrm{U}}$ of the cache-enabled ground SBSs and UAVs, respectively. These SBSs and UAVs can 
support the typical user when the $k$-th content is requested, and $\Phi_{\mathrm{G}}=\cup_{k \in K} \Phi_{\mathrm{G}, k}$, and $\Phi_{\mathrm{U}}=\cup_{k \in K} \Phi_{\mathrm{U}, k}$, respectively. All the remaining un-cached contents having least popularity are served by the macro BS via backhaul.

\section{INTERFERENCE CHARACTERIZATION}

Considering the downlink communication, we focus on the scenario where UAVs establish a communication link with the typical user with the strongest received signal and consequently provide the highest SINR for the user. Thus, the received SINR at the typical user is given by

$$
\mathrm{SINR}_{\mathrm{U}, \mathrm{z}}=\frac{P_{\mathrm{U}} g_{o}\left\{L_{\mathrm{U}, \mathrm{z}}\left(X_{o}\right)\right\}^{-1}}{\sigma^{2}+\mathcal{I}_{\mathrm{U}}+\hat{\mathcal{I}}_{\mathrm{G}}} ; \quad \mathrm{z} \in\{\mathrm{L}, \mathrm{N}\},
$$

where $X_{o}$ denotes the distance from the typical user to its serving UAV, $g_{o} \sim \exp (1)$ is the Rayleigh fading channel power with unit mean, $\sigma^{2}$ is the noise power, and the aggregate intra-tier interference is given by $\mathcal{I}_{\mathrm{U}}=\mathcal{I}_{\mathrm{U}, \mathrm{C}}+\mathcal{I}_{\mathrm{U}, \mathrm{N}}$ with

$$
\mathcal{I}_{\mathrm{U}, \mathrm{C}}=\sum_{\mathrm{z} \in\{\mathrm{L}, \mathrm{N}\}} \sum_{i \in \Phi_{\mathrm{U}, k} \backslash\{o\}} P_{\mathrm{U}} g_{i}\left\{L_{\mathrm{U}, \mathrm{Z}}\left(X_{i}\right)\right\}^{-1},
$$

and

$$
\mathcal{I}_{\mathrm{U}, \mathrm{N}}=\sum_{\mathrm{z} \in\{\mathrm{L}, \mathrm{N}\}} \sum_{j \in \Phi_{\mathrm{U}} \backslash \Phi_{\mathrm{U}, k}} P_{\mathrm{U}} g_{j}\left\{L_{\mathrm{U}, \mathrm{Z}}\left(X_{j}\right)\right\}^{-1},
$$

being the co-channel interferences from the UAVs with the propagation distance $X_{i}$ and the point process $\Phi_{\mathrm{U}, k} \backslash\{o\}$ corresponding to the density $b_{k} \lambda_{\mathrm{U}}$ that cache the $k$-th content, and from the UAVs with the propagation distance $X_{j}$ and the point process $\Phi_{\mathrm{U}} \backslash \Phi_{\mathrm{U}, k}$ corresponding to the density given by independent thinning theorem $\left(1-b_{k}\right) \lambda_{\mathrm{U}}$ [30] that do not store the $k$-th content in their caches, respectively. Also, the aggregate inter-tier interference caused by the ground SBSs in tier $\mathrm{G}$ is given by

$$
\hat{\mathcal{I}}_{\mathrm{G}}=\sum_{l \in \Phi_{\mathrm{G}}} P_{\mathrm{G}} g_{l}\left\{L_{\mathrm{G}}\left(Y_{l}\right)\right\}^{-1},
$$

where $Y_{l}$ is the propagation distance between the typical user and the ground SBS, $g_{i}, g_{j}, g_{l} \sim \exp (1)$ are the interfering Rayleigh channel fading powers that follow the exponential distribution.

Similarly, the received SINR at the typical user from the ground SBSs in tier $\mathrm{G}$ is given by

$$
\operatorname{SINR}_{\mathrm{G}}=\frac{P_{\mathrm{G}} g_{o}\left\{L_{\mathrm{G}}\left(Y_{o}\right)\right\}^{-1}}{\sigma^{2}+\mathcal{I}_{\mathrm{G}}+\hat{\mathcal{I}}_{\mathrm{U}}},
$$

where the aggregate intra-tier interference is given by $\mathcal{I}_{\mathrm{G}}=$ $\mathcal{I}_{\mathrm{G}, \mathrm{C}}+\mathcal{I}_{\mathrm{G}, \mathrm{N}}$ with

$$
\mathcal{I}_{\mathrm{G}, \mathrm{C}}=\sum_{i \in \Phi_{\mathrm{G}, k} \backslash\{o\}} P_{\mathrm{G}} g_{i}\left\{L_{\mathrm{G}}\left(Y_{i}\right)\right\}^{-1},
$$

and

$$
\mathcal{I}_{\mathrm{G}, \mathrm{N}}=\sum_{j \in \Phi_{\mathrm{G}} \backslash \Phi_{\mathrm{G}, k}} P_{\mathrm{G}} g_{j}\left\{L_{\mathrm{G}}\left(Y_{j}\right)\right\}^{-1},
$$

being the intra-tier interferences from the ground SBSs with the ground distance $Y i$ and the point process $\Phi_{\mathrm{G}, k} \backslash\{o\}$ corresponding to the density $b_{k} \lambda_{\mathrm{G}}$ that store the $k$-th content, and from the ground SBSs with the ground distance $Y j$ and the point process $\Phi_{\mathrm{G}} \backslash \Phi_{\mathrm{G}, k}$ corresponding to the density $\left(1-b_{k}\right) \lambda_{\mathrm{G}}$ that do not store the $k$-th file. Also, the aggregate inter-tier interference caused by all UAVs in tier $U$ is given by

$$
\hat{\mathcal{I}}_{\mathrm{U}}=\sum_{\mathrm{z} \in\{\mathrm{L}, \mathrm{N}\}} \sum_{l \in \Phi_{\mathrm{U}}} P_{\mathrm{U}} g_{l}\left\{L_{\mathrm{U}, \mathrm{z}}\left(X_{l}\right)\right\}^{-1} .
$$

where $X_{l}$ is the interfering propagation distance from UAVs in tier $\mathrm{U}$.

\section{Derivation of User Association Probability}

User association accounts for both the content availability and the link reliability. Therefore, we introduce a user association method based on the maximum received signal power by the user from the UAV $\left(\mathbb{L}_{\mathrm{U}, x}\right)$ and the ground SBS $\left(\mathbb{L}_{\mathrm{G}, y}\right)$ with the following criterion

$$
\mathbb{L}_{\mathrm{U}, x}=P_{\mathrm{U}} g_{o}\left(\sum_{\mathrm{z} \in\{\mathrm{L}, \mathrm{N}\}} L_{\mathrm{U}, \mathrm{z}}\left(r_{x}\right)^{-1} p_{\mathrm{z}}\left(r_{x}\right)\right),
$$

and

$$
\mathbb{L}_{\mathrm{G}, y}=P_{\mathrm{G}} g_{o} L_{\mathrm{G}}\left(r_{y}\right)^{-1} \text {. }
$$

It is important to note that only the popular parts of all contents, i.e., $\mathcal{C}^{\mathrm{U}}$ and $\mathcal{C}^{\mathrm{G}}$, are cached at the UAV and ground SBSs due to their limited storage capacity. Hence, it is possible that a file requested by the user may be unavailable at SBSs, which has to be requested via the backhaul. However, such situation arises occasionally and are omitted in our analysis for mathematical tractability, similar to [36], [37]. A user is associated with the content-centric SBSs if the user requests file $f_{k}$ and is served by UAV in tier U and SBS in tier G. Thus, when the user requests the file $f_{k}$, the serving UAV and SBS denoted by $\mathbb{L}_{k}$ is defined as

$$
\mathbb{L}_{k}=\left\{\begin{array}{l}
\arg \max \left\{\max _{x \in \Phi_{\mathrm{U}, k}} \mathbb{L}_{\mathrm{U}, x}, \max _{y \in \Phi_{\mathrm{G}, k}} \mathbb{L}_{\mathrm{G}, y}\right\}, f_{k} \in\left\{\mathcal{C}^{\mathrm{U}}, \mathcal{C}^{\mathrm{G}}\right\} \\
\arg \max _{x \in \Phi_{\mathrm{U}, k}} \mathbb{L}_{\mathrm{U}, x}, f_{k} \in \mathcal{C}^{\mathrm{U}} \\
\arg \max _{y \in \Phi_{\mathrm{G}, k}} \mathbb{L}_{\mathrm{G}, y}, f_{k} \in \mathcal{C}^{\mathrm{G}} .
\end{array}\right.
$$

where we assume fixed $P_{\mathrm{U}}$ in tier $\mathrm{U}$ and $P_{\mathrm{G}}$ in tier $\mathrm{G}$, respectively. In this case, the variable transmit power of UAVs may not be useful due to the properties of homogeneous PPP and the user association scheme in (15).

Lemma 1: The probability that a typical user is associated with the nearest cache-enabled UAV with file $f_{k}$ in LOS and NLOS conditions is given by

$$
\mathcal{A}_{\mathrm{U}}^{k}=\sum_{\mathrm{z} \in\{\mathrm{L}, \mathrm{N}\}} \mathcal{A}_{\mathrm{U}, \mathrm{z}}^{k}
$$

where, $\mathcal{A}_{\mathrm{U}, \mathrm{L}}^{k}$ and $\mathcal{A}_{\mathrm{U}, \mathrm{N}}^{k}$ are association probabilities for LOS and NLOS conditions, respectively, and $\mathcal{A}_{\mathrm{U}, \mathrm{L}}^{k}$ is calculated as

$$
\begin{gathered}
\mathcal{A}_{\mathrm{U}, \mathrm{L}}^{k}=2 \pi b_{k} \lambda_{\mathrm{U}} \int_{h}^{\infty} r_{x} \exp \left(-\pi b_{k} \lambda_{\mathrm{G}}\left(\frac{P_{\mathrm{G}}}{P_{\mathrm{U}}} r_{x} \alpha_{\mathrm{L}}\right)^{2 / \alpha_{\mathrm{G}}}\right. \\
\left.-2 \pi b_{k} \lambda_{\mathrm{U}} \int_{h}^{r_{x}} p_{\mathrm{L}}(l) l d l\right) p_{\mathrm{L}}\left(r_{x}\right) d r_{x}
\end{gathered}
$$


Proof: Assume that $r_{x}$ is the minimum propagation distance between the UAV with file $f_{k}$ in tier $\mathrm{U}$ to the typical user and $r_{y}$ is the distance from the ground SBS in tier G to the typical user. Thus, the user association probability for the UAV in the LOS condition $\mathcal{A}_{\mathrm{U}, \mathrm{L}}^{k}$ is the probability that $L_{\mathrm{U}, \mathrm{L}}\left(r_{x}\right)>$ $L_{\mathrm{G}}\left(r_{y}\right)$. Therefore,

$$
\begin{aligned}
\mathcal{A}_{\mathrm{U}, \mathrm{L}}^{k} & =\mathbb{E}_{r_{x}}\left[\mathbb{P}\left[L_{\mathrm{U}, \mathrm{L}}\left(r_{x}\right)>L_{\mathrm{G}}\left(r_{y}\right)\right]\right] \\
& =\int_{h}^{\infty} \mathbb{P}\left[r_{y}>\left(\frac{P_{\mathrm{G}}}{P_{\mathrm{U}}} r_{x}^{\alpha_{\mathrm{L}}}\right)^{1 / \alpha_{\mathrm{G}}}\right] f_{r_{x}}^{\mathrm{L}}\left(r_{x}\right) d r_{x} .
\end{aligned}
$$

where $\mathbb{P}\left[r_{y}>\left(\frac{P_{\mathrm{G}}}{P_{\mathrm{U}}} r_{x}^{\alpha_{\mathrm{L}}}\right)^{1 / \alpha_{\mathrm{G}}}\right]$ is computed by using the null probability of a $2 \mathrm{D}$ Poisson process as

$$
\begin{aligned}
\mathbb{P}\left[r_{y}>\right. & \left.\left(\frac{P_{\mathrm{G}}}{P_{\mathrm{U}}} r_{x}^{\alpha_{\mathrm{L}}}\right)^{1 / \alpha_{\mathrm{G}}}\right]= \\
& \exp \left(-\pi b_{k} \lambda_{\mathrm{G}}\left(\frac{P_{\mathrm{G}}}{P_{\mathrm{U}}} r_{x}{ }^{\alpha_{\mathrm{L}}}\right)^{2 / \alpha_{\mathrm{G}}}\right) .
\end{aligned}
$$

Furthermore, the PDF of $r_{x}$ denoted by $f_{r_{x}}^{\mathrm{L}}\left(r_{x}\right)$ corresponds to the serving UAVs in LOS condition with probability $p_{\mathrm{L}}\left(r_{x}\right)$ that provides strongest signal to the typical user with the shortest distance. In this case, $f_{r_{x}}^{\mathrm{L}}\left(r_{x}\right)$ is derived by taking the derivative of $1-\mathbb{P}\left[h<l<r_{x}\right]$ with respect to $r_{x}$ and using the null probability of a $2 \mathrm{D}$ Poisson process, where

$$
\mathbb{P}\left[h<l<r_{x}\right]=\exp \left(-2 \pi b_{k} \lambda_{\mathrm{U}} \int_{h}^{r_{x}} p_{\mathrm{L}}(l) l d l\right) .
$$

and $l$ is the minimum propagation distance range. Then, by using Leibniz integral rule, $f_{r_{x}}^{\mathrm{L}}\left(r_{x}\right)$ is given by

$$
\begin{aligned}
f_{r_{x}}^{\mathrm{L}}\left(r_{x}\right) & =2 \pi r_{x} b_{k} \lambda_{\mathrm{U}} p_{\mathrm{L}}\left(r_{x}\right) \\
& \times \exp \left(-2 \pi b_{k} \lambda_{\mathrm{U}} \int_{h}^{r_{x}} p_{\mathrm{L}}(l) l d l\right) .
\end{aligned}
$$

Substituting (19) and (21) in (18), we obtain the result in (17). Similarly, the association probability $\mathcal{A}_{\mathrm{U}, \mathrm{N}}^{k}$ for the UAV in the NLOS condition can be derived by following the same steps as

$$
\begin{aligned}
\mathcal{A}_{\mathrm{U}, \mathrm{N}}^{k}= & 2 \pi b_{k} \lambda_{\mathrm{U}} \int_{h}^{\infty} r_{x} \exp \left(-\pi b_{k} \lambda_{\mathrm{G}}\left(\frac{P_{\mathrm{G}}}{P_{\mathrm{U}}} r_{x}{ }^{\alpha_{\mathrm{N}}}\right)^{2 / \alpha_{\mathrm{G}}}\right. \\
& \left.-2 \pi b_{k} \lambda_{\mathrm{U}} \int_{h}^{r_{x}} p_{\mathrm{N}}(l) l d l\right) p_{\mathrm{N}}\left(r_{x}\right) d r_{x}
\end{aligned}
$$

Lemma 2: The probability that a typical user is associated with the nearest cache-enabled ground SBS with file $f_{k}$ is given by

$$
\begin{aligned}
\mathcal{A}_{\mathrm{G}}= & 2 \pi b_{k} \lambda_{\mathrm{G}} \sum_{\mathrm{z} \in\{\mathrm{L}, \mathrm{N}\}} \int_{0}^{\infty} r_{y} \exp \left(-\pi b_{k} \lambda_{\mathrm{G}} r_{y}{ }^{2}\right. \\
& \left.-2 \pi b_{k} \lambda_{\mathrm{U}} \int_{h}^{\left(\frac{P_{\mathrm{U}}}{P_{\mathrm{G}}} r_{y}^{\alpha_{\mathrm{G}}}\right)^{2 / \alpha_{\mathrm{z}}}} p_{\mathrm{z}}\left(r_{x}\right) r_{x} d r_{x}\right) d r_{y}
\end{aligned}
$$

Proof: Assume that the minimum distance between the ground SBS with file $f_{k}$ in tier $\mathrm{G}$ to the typical user is $r_{y}$, and the propagation distance between the UAV in tier $U$ to the typical user is $r_{x}$. Thus,

$$
\begin{array}{r}
\mathcal{A}_{G}=\mathbb{E}_{r_{y}}\left[\mathbb{P}\left[L_{\mathrm{G}}\left(r_{y}\right)>L_{\mathrm{U}, \mathrm{z}}\left(r_{x}\right)\right]\right] \\
=\int_{0}^{\infty} \mathbb{P}\left[h<r_{x}<\left(\frac{P_{\mathrm{U}}}{P_{\mathrm{G}}} r_{y}^{\alpha_{\mathrm{G}}}\right)^{1 / \alpha_{\mathrm{z}}}\right] f_{r_{y}}^{\mathrm{G}}\left(r_{y}\right) d r_{y} .
\end{array}
$$

Then using null probability of a 2D Poisson process, we have

$$
\begin{array}{r}
\mathbb{P}\left[h<r_{x}<\left(\frac{P_{\mathrm{U}}}{P_{\mathrm{G}}} r_{y}^{\alpha_{\mathrm{G}}}\right)^{1 / \alpha_{\mathrm{z}}}\right]= \\
\exp \left(-2 \pi b_{k} \lambda_{\mathrm{U}} \int_{h}^{\left(\frac{P_{\mathrm{U}}}{P_{\mathrm{G}}} r_{y}^{\alpha_{\mathrm{G}}}\right)^{1 / \alpha_{\mathrm{Z}}}} p_{\mathrm{z}}\left(r_{x}\right) r_{x} d r_{x}\right) .
\end{array}
$$

The PDF of the minimum ground distance $r_{y}$ is given by

$$
f_{r_{y}}^{\mathrm{G}}\left(r_{y}\right)=2 \pi b_{k} \lambda_{\mathrm{G}} r_{y} \exp \left(-\pi b_{k} \lambda_{\mathrm{G}} r_{y}^{2}\right) .
$$

Substituting (25) and (27) in (24), we obtain the result in (23).

\section{The Successful Content Delivery Probability}

The network performance is measured by the successful content delivery probability, which represents the probability that the file requested by a typical user is not only cached at UAV and SBS but also successfully transmitted by them over the wireless channel. For the density share scheme, the successful content delivery probability of the considered hybrid network is given by

$$
\mathcal{P}_{\mathrm{SCD}}\left(\eta, b_{k}\right)=\mathcal{P}_{\mathrm{SCD}}^{\mathrm{U}}\left(\eta, b_{k}\right)+\mathcal{P}_{\mathrm{SCD}}^{\mathrm{G}}\left(\eta, b_{k}\right),
$$

assuming that the content size of $\varphi$ bits needs to be transmitted in $\tau$ seconds. One has

$$
\mathcal{P}_{\mathrm{SCD}}^{\mathrm{U}}\left(\eta, b_{k}\right)=\sum_{k=1}^{J} m_{k} \mathbb{P}\left[\operatorname{SINR}_{\mathrm{U}, \mathrm{z}}>\delta_{\mathrm{U}}\right]
$$

with $\delta_{\mathrm{U}}=2^{\frac{\varphi / \tau}{\mathcal{W}_{\mathrm{U}}}}-1$ and $\mathcal{W}_{\mathrm{U}}$ being the UAV bandwidth and

$$
\mathcal{P}_{\mathrm{SCD}}^{\mathrm{G}}\left(\eta, b_{k}\right)=\sum_{k=1}^{J} m_{k} \mathbb{P}\left[\operatorname{SINR}_{\mathrm{G}}>\delta_{\mathrm{G}}\right],
$$

where $\delta_{\mathrm{G}}=2^{\frac{\varphi / \tau}{\mathcal{W}_{\mathrm{G}}}}-1$, and $\mathcal{W}_{\mathrm{G}}$ is the ground SBS bandwidth.

\section{A. Successful Content Delivery Probability for the UAV in tier $\mathrm{U}$}

Lemma 3: The successful content delivery probability for the cache-enabled UAV with file $f_{k}$ in tier $\mathrm{U}$ is given by

$$
\mathcal{P}_{\mathrm{SCD}}^{\mathrm{U}}\left(\eta, b_{k}\right)=\sum_{k=1}^{J} m_{k} \mathcal{P}_{\mathrm{Cov}}^{\mathrm{U}}\left(\eta, b_{k}\right)
$$

where $P_{\mathrm{Cov}}^{\mathrm{U}}\left(\eta, b_{k}\right)=P_{\mathrm{Cov}}^{\mathrm{U}, \mathrm{L}}\left(\eta, b_{k}\right)+P_{\mathrm{Cov}}^{\mathrm{U}, \mathrm{N}}\left(\eta, b_{k}\right)$ and $\mathcal{P}_{\mathrm{Cov}}^{\mathrm{U}, \mathrm{L}}\left(\eta, b_{k}\right)$ and $\mathcal{P}_{\mathrm{Cov}}^{\mathrm{U}, \mathrm{N}}\left(\eta, b_{k}\right)$ are the coverage probabilities to successfully delivered the file $f_{k}$ with caching probability $b_{k}$ to a typical user connected with the serving UAV in tier U with density $\lambda_{\mathrm{U}}=\eta \lambda_{\mathrm{S}}$ having LOS and NLOS conditions, respectively, and are given as

$$
\begin{aligned}
& \mathcal{P}_{\mathrm{Cov}}^{\mathrm{U}, \mathrm{L}}\left(\eta, b_{k}\right)= \\
& \mathcal{L}_{\mathcal{I}_{\mathrm{U}, \mathrm{C}}}\left(s_{\mathrm{L}}\right) \cdot \mathcal{L}_{\mathcal{I}_{\mathrm{U}, \mathrm{N}}}\left(s_{\mathrm{L}}\right) \cdot \mathcal{L}_{\hat{\mathcal{I}}_{\mathrm{G}}}\left(s_{\mathrm{L}}\right) \cdot \exp \left(-s_{\mathrm{L}} \sigma^{2}\right) \cdot \mathcal{A}_{\mathrm{U}, \mathrm{L}}^{k},
\end{aligned}
$$




$$
\begin{aligned}
& \mathcal{P}_{\mathrm{Cov}}^{\mathrm{U}, \mathrm{N}}\left(\eta, b_{k}\right)= \\
& \mathcal{L}_{\mathcal{I}_{\mathrm{U}, \mathrm{C}}}\left(s_{\mathrm{N}}\right) \cdot \mathcal{L}_{\mathcal{I}_{\mathrm{U}, \mathrm{N}}}\left(s_{\mathrm{N}}\right) \cdot \mathcal{L}_{\hat{\mathcal{I}}_{\mathrm{G}}}\left(s_{\mathrm{N}}\right) \cdot \exp \left(-s_{\mathrm{N}} \sigma^{2}\right) \cdot \mathcal{A}_{\mathrm{U}, \mathrm{N}}^{k},
\end{aligned}
$$

respectively, where $s_{\mathrm{z}}=\frac{\delta_{\mathrm{U}} \beta_{\mathrm{o}}}{P_{\mathrm{U}} \sqrt{y^{2}+h^{2}}-\alpha_{\mathrm{z}}}$ for $\mathrm{z} \in\{\mathrm{L}, \mathrm{N}\}$, $\mathcal{A}_{\mathrm{U}, \mathrm{L}}^{k}$, and $\mathcal{A}_{\mathrm{U}, \mathrm{N}}^{k}$ are given in (17) and (22), respectively. Also, $\mathcal{L}_{\mathcal{L}_{U}, \mathrm{C}}(\cdot), \mathcal{L}_{\mathcal{I}_{U, \mathrm{~N}}}(\cdot)$, and $\mathcal{L}_{\hat{\mathcal{I}}_{\mathrm{G}}}(\cdot)$ are the Laplace transforms of the interference generated by UAVs that cache with file $f_{k}$, UAVs that do not cache $f_{k}$, and ground network, respectively.

Proof: Given that a typical user is associated with the cacheenabled UAV in tier $U$ in the LOS condition, the connection probability in the presence of the intra-tier interference from UAVs that cache and do not cache $f_{k}$ and inter-tier interference from the ground network is given as

$$
\begin{aligned}
& \mathcal{P}_{\mathrm{Cov}}^{\mathrm{U}, \mathrm{L}}\left(\eta, b_{k}\right)=\mathbb{P}\left[\mathrm{SINR}_{\mathrm{U}, \mathrm{L}}>\delta_{\mathrm{U}}\right] \\
& =\mathbb{P}\left[g_{o}>\frac{\delta_{\mathrm{U}} L_{\mathrm{U}, \mathrm{z}}}{P_{\mathrm{U}}}\left(\sigma^{2}+\mathcal{I}_{\mathrm{U}, \mathrm{C}}+\mathcal{I}_{\mathrm{U}, \mathrm{N}}+\hat{\mathcal{I}}_{\mathrm{G}}\right)\right] \\
& =\exp \left(-s_{\mathrm{L}} \sigma^{2}\right) \cdot \mathcal{L}_{\mathcal{I}_{\mathrm{U}, \mathrm{C}}}\left(s_{\mathrm{L}}\right) \cdot \mathcal{L}_{\mathcal{I}_{\mathrm{U}, \mathrm{N}}}\left(s_{\mathrm{L}}\right) \cdot \mathcal{L}_{\hat{\mathcal{I}}_{\mathrm{G}}}\left(s_{\mathrm{L}}\right),
\end{aligned}
$$

where $s_{\mathrm{L}}=\frac{\delta_{\mathrm{U}} \beta_{\mathrm{o}}}{P_{\mathrm{U}} \sqrt{y^{2}+h^{2}}-\alpha_{\mathrm{L}}}$. Properties of the stochastic geometry can be used to determine the Laplace transforms. Thus, $\mathcal{L}_{\mathcal{I}_{\mathrm{U}, \mathrm{C}}}\left(s_{\mathrm{L}}\right)$ is given by

$$
\begin{aligned}
& \mathcal{L}_{\mathcal{I}_{\mathrm{U}, \mathrm{C}}}\left(s_{\mathrm{L}}\right)=\mathbb{E}_{\Phi_{\mathrm{U}, k} \backslash\{o\}}\left[\exp \left(-s_{\mathrm{L}} \mathcal{I}_{\mathrm{U}, \mathrm{C}}\right)\right] \\
& \stackrel{(a)}{=} \exp \left(-2 \pi b_{k} \lambda_{\mathrm{U}} \sum_{\mathrm{z} \in\{\mathrm{L}, \mathrm{N}\}} \int_{y}^{\infty}(1-\right. \\
& \left.\left.\mathbb{E}_{g_{i}}\left[\exp \left(\frac{-s_{\mathrm{L}} P_{\mathrm{U}} g_{i}{\sqrt{x^{2}+h^{2}}}^{-\alpha_{\mathrm{z}}}}{\beta_{\mathrm{O}}}\right)\right]\right) x p_{\mathrm{z}}\left(x^{\prime}\right) d x\right) \\
& \stackrel{(b)}{=} \exp \left(-2 \pi b_{k} \lambda_{\mathrm{U}} \times\right. \\
& \left.\sum_{\mathrm{z} \in\{\mathrm{L}, \mathrm{N}\}} \int_{y}^{\infty}\left(\frac{s_{\mathrm{L}} P_{\mathrm{U}}{\sqrt{x^{2}+h^{2}}}^{-\alpha_{\mathrm{z}}}}{\beta_{\mathrm{O}}+s_{\mathrm{L}} P_{\mathrm{U}}{\sqrt{x^{2}+h^{2}}}^{-\alpha_{\mathrm{z}}}}\right) x p_{\mathrm{z}}\left(x^{\prime}\right) d x\right),
\end{aligned}
$$

where $x^{\prime}=\sqrt{x^{2}+h^{2}}$, (a) comes from the probability generating functional of PPP and (b) follows the moment generating function of the exponential distribution [21]. Likewise, $\mathcal{L}_{\mathcal{I}_{\mathrm{U}, \mathrm{N}}}\left(s_{\mathrm{L}}\right)$ is given as

$$
\begin{aligned}
& \mathcal{L}_{\mathcal{I}_{\mathrm{U}, \mathrm{N}}}\left(s_{\mathrm{L}}\right)=\exp \left(-2 \pi\left(1-b_{k}\right) \lambda_{\mathrm{U}} \times\right. \\
& \left.\sum_{\mathrm{z} \in\{\mathrm{L}, \mathrm{N}\}} \int_{y}^{\infty}\left(\frac{s_{\mathrm{L}} P_{\mathrm{U}}{\sqrt{x^{2}+h^{2}}}^{-\alpha_{\mathrm{z}}}}{\beta_{\mathrm{O}}+s_{\mathrm{L}} P_{\mathrm{U}}{\sqrt{x^{2}+h^{2}}}^{-\alpha_{\mathrm{z}}}}\right) x p_{\mathrm{Z}}\left(x^{\prime}\right) d x\right) .
\end{aligned}
$$

The Laplace transform for interference from the ground network is given as

$$
\begin{aligned}
& \mathbb{E}_{\Phi_{\mathrm{G}}}\left[\exp \left(-s_{\mathrm{L}} \hat{\mathcal{I}}_{\mathrm{G}}\right)\right] \\
& =\exp \left(-2 \pi \lambda_{\mathrm{G}} \int_{0}^{\infty}\left(1-\frac{1}{1+\frac{s_{\mathrm{L}} P_{\mathrm{G}} r^{-\alpha_{\mathrm{G}}}}{\beta_{\mathrm{o}}}}\right) x d x\right) \\
& =\exp \left(-2 \pi \lambda_{\mathrm{G}} \int_{0}^{\infty} \frac{\delta_{\mathrm{U}} P_{\mathrm{G}}{\sqrt{y^{2}+h^{2}}}^{\alpha_{\mathrm{L}}}}{P_{\mathrm{U}} r^{\alpha_{\mathrm{G}}}+\delta_{\mathrm{U}} P_{\mathrm{G}}{\sqrt{y^{2}+h^{2}}}^{\alpha_{\mathrm{L}}}} x d x\right), \\
& =\exp \left(-2 \pi^{2} \lambda_{\mathrm{G}} \frac{\delta_{\mathrm{U}}^{2 / \alpha_{\mathrm{G}}}\left(y^{2}+h^{2}\right)^{\alpha_{\mathrm{L}} / \alpha_{\mathrm{G}}} \csc \left(\frac{2 \pi}{\alpha_{\mathrm{G}}}\right)}{\alpha_{\mathrm{G}}\left(\frac{P_{\mathrm{U}}}{P_{\mathrm{G}}}\right)^{2 / \alpha_{\mathrm{G}}}}\right) .
\end{aligned}
$$

where $\csc (\cdot)$ is the Cosecant trigonometry function.

Similarly, Laplace transforms of cached and un-cached UAVs in NLOS condition can be computed by following the same steps. Next we will derive the successful content delivery probability for the ground SBS in tier G.

\section{B. Successful Content Delivery Probability for the SBS in tier $\mathrm{G}$}

Lemma 4: The successful content delivery probability for the cache-enabled ground SBS with file $f_{k}$ in tier G is given by

$$
\mathcal{P}_{\mathrm{SCD}}^{\mathrm{G}}\left(\eta, b_{k}\right)=\sum_{k=1}^{J} m_{k} \mathcal{P}_{, \mathrm{Cov}}^{\mathrm{G}}\left(\eta, b_{k}\right),
$$

where $\mathcal{P}_{\text {Cov }}^{\mathrm{G}}\left(\eta, b_{k}\right)$ is the coverage probability to successfully deliver the file $f_{k}$ with caching probability $b_{k}$ to the ground user connected with the serving ground SBS in tier G with SBS density $\lambda_{\mathrm{G}}=(1-\eta) \lambda_{\mathrm{S}}$ and is given as

$$
\begin{aligned}
& \mathcal{P}_{\mathrm{Cov}}^{\mathrm{G}}\left(\eta, b_{k}\right)=\mathbb{P}\left[\operatorname{SINR}_{\mathrm{G}}>\delta_{\mathrm{G}}\right] \\
& =\mathbb{P}\left[g_{o}>\frac{\delta_{\mathrm{G}} L_{\mathrm{G}}}{P_{\mathrm{G}}}\left(\sigma^{2}+\mathcal{I}_{\mathrm{G}, \mathrm{C}}+\mathcal{I}_{\mathrm{G}, \mathrm{N}}+\hat{\mathcal{I}}_{\mathrm{U}}\right)\right] \\
& =\mathcal{L}_{\mathcal{I}_{\mathrm{G}, \mathrm{C}}}\left(s_{\mathrm{G}}\right) \cdot \mathcal{L}_{\mathcal{I}_{\mathrm{G}, \mathrm{N}}}\left(s_{\mathrm{G}}\right) \cdot \mathcal{L}_{\hat{\mathcal{I}}_{\mathrm{U}}}\left(s_{\mathrm{G}}\right) \cdot \exp \left(-s_{\mathrm{G}} \sigma^{2}\right) \cdot \mathcal{A}_{\mathrm{G}},
\end{aligned}
$$

where $s_{\mathrm{G}}=\frac{\delta_{\mathrm{G}} \beta_{\mathrm{o}}}{P_{\mathrm{G}} x^{-\alpha_{\mathrm{G}}}}, \mathcal{A}_{\mathrm{G}}$ is given in (23), and $\mathcal{L}_{\mathcal{I}_{\mathrm{G}, \mathrm{C}}}(\cdot)$, $\mathcal{L}_{\mathcal{I}_{\mathrm{G}, \mathrm{N}}}(\cdot)$ and $\mathcal{L}_{\hat{\mathcal{I}}_{\mathrm{U}}}(\cdot)$ are the Laplace transforms of the intratier interference generated by ground SBSs that store $f_{k}$, ground SBSs that do not store $f_{k}$, and the UAV network, respectively.

Proof: The Laplace transform for the intra-tier interference generated by the ground SBSs that cache the file $f_{k}$ is given as

$$
\begin{aligned}
& \mathcal{L}_{\mathcal{I}_{\mathrm{G}, \mathrm{C}}}\left(s_{\mathrm{G}}\right)=\mathbb{E}_{\Phi_{\mathrm{G}, k} \backslash\{o\}}\left[\exp \left(-s_{\mathrm{G}} \mathcal{I}_{\mathrm{G}, \mathrm{C}}\right)\right] \\
& =\exp \left(-2 \pi b_{k} \lambda_{\mathrm{G}} \int_{y}^{\infty} \times\right. \\
& \left.\left(1-\mathbb{E}_{g_{i}}\left[\exp \left(-\frac{s_{\mathrm{G}} P_{\mathrm{G}} g_{i} x^{-\alpha_{\mathrm{G}}}}{\beta_{\mathrm{o}}}\right)\right]\right) x d x\right) \\
& =\exp \left(-2 \pi b_{k} \lambda_{\mathrm{G}} \int_{y}^{\infty}\left(\frac{s_{\mathrm{G}} P_{\mathrm{G}} r^{-\alpha_{\mathrm{G}}}}{\beta_{\mathrm{o}}+s_{\mathrm{G}} P_{\mathrm{G}} x^{-\alpha_{\mathrm{G}}}}\right) x d x\right) \\
& =\exp \left(-2 \pi b_{k} \lambda_{\mathrm{G}} \frac{\delta_{\mathrm{G}} y^{2}}{\alpha_{\mathrm{G}}-2} \times_{2} F_{1}\left(1,1-\frac{2}{\alpha_{\mathrm{G}}}, 2-\frac{2}{\alpha_{\mathrm{G}}},-\delta_{\mathrm{G}}\right)\right) .
\end{aligned}
$$


where ${ }_{2} F_{1}(\cdot, \cdot, \cdot, \cdot)$ is the Gauss hypergeometric function. Likewise, $\mathcal{L}_{\mathcal{I}_{\mathrm{G}, \mathrm{N}}}\left(s_{\mathrm{G}}\right)$ is given as

$$
\begin{aligned}
\mathcal{L}_{\mathcal{I}_{\mathrm{G}, \mathrm{N}}}\left(s_{\mathrm{G}}\right) & =\exp \left(-2 \pi\left(1-b_{k}\right) \lambda_{\mathrm{G}} \frac{\delta_{\mathrm{G}} y^{2}}{\alpha_{\mathrm{G}}-2}\right. \\
& \left.{ }_{2} F_{1}\left(1,1-\frac{2}{\alpha_{\mathrm{G}}}, 2-\frac{2}{\alpha_{\mathrm{G}}},-\delta_{\mathrm{G}}\right)\right) .
\end{aligned}
$$

Using the proof of Lemma $3, \mathcal{L}_{\hat{\mathcal{I}}_{\mathrm{U}}}\left(s_{\mathrm{G}}\right)$ is given as

$$
\begin{aligned}
& \mathcal{L}_{\hat{\mathcal{I}}_{\mathrm{U}}}\left(s_{\mathrm{G}}\right)=\exp \left(-2 \pi \lambda_{\mathrm{U}} \times\right. \\
& \left.\sum_{\mathrm{z} \in\{\mathrm{L}, \mathrm{N}\}} \int_{y}^{\infty}\left(\frac{s_{\mathrm{G}} P_{\mathrm{U}}{\sqrt{x^{2}+h^{2}}}^{-\alpha_{\mathrm{z}}}}{\beta_{\mathrm{o}}+s_{\mathrm{G}} P_{\mathrm{U}}{\sqrt{x^{2}+h^{2}}}^{-\alpha_{\mathrm{z}}}}\right) x p_{\mathrm{z}}\left(x^{\prime}\right) d x\right) .
\end{aligned}
$$

\section{ENERgy EFFICIENCy of Hybrid Network}

Energy efficiency is a widely used performance measure for the heterogeneous network with dense and random deployment of SBSs. Using (30) and (37), we have obtained the successful content delivery probabilities for the UAV and ground networks, respectively. In this case, the throughput attained at a typical user by the UAV and ground SBS are given by $\sum_{k=1}^{J} m_{k} \mathbb{P}\left[\operatorname{SINR}_{\mathrm{U}, \mathrm{z}}>\delta_{\mathrm{U}}\right] \log _{2}\left(1+\delta_{\mathrm{U}}\right)$ and $\sum_{k=1}^{J} m_{k} \mathbb{P}\left[\operatorname{SINR}_{\mathrm{G}}>\delta_{\mathrm{G}}\right] \log _{2}\left(1+\delta_{\mathrm{G}}\right)$, respectively, and the area spectral efficiency is taken over the UAV-user and ground SBS-user links in the network. Thus, for the UAV and ground homogeneous network, the area spectral efficiency is defined as $\lambda_{\mathrm{U}} \mathcal{P}_{\mathrm{SCD}}^{\mathrm{U}}\left(\eta, b_{k}\right) \log _{2}\left(1+\delta_{\mathrm{U}}\right)$ and $\lambda_{\mathrm{G}} \mathcal{P}_{\mathrm{SCD}}^{\mathrm{G}}\left(\eta, b_{k}\right) \log _{2}(1+$ $\left.\delta_{\mathrm{G}}\right)$, respectively. Finally, we define the energy efficiency of the hybrid network as [32]

$$
\mathrm{EE}=\frac{\left[\lambda_{\mathrm{U}} \mathcal{P}_{\mathrm{SCD}}^{\mathrm{U}}\left(\eta, b_{k}\right)+\lambda_{\mathrm{G}} \mathcal{P}_{\mathrm{SCD}}^{\mathrm{G}}\left(\eta, b_{k}\right)\right] \log _{2}(1+\delta)}{\lambda_{\mathrm{U}}\left(P_{\mathrm{U}}+P_{\mathrm{hov}}\right)+\lambda_{\mathrm{G}}\left(P_{\mathrm{G}}+\triangle_{\mathrm{G}} P_{\mathrm{RF}}\right)},
$$

where $\delta$ is a prescribed quality-of-service requirement. The UAVs in our work are static during transmission and therefore, $P_{\text {hov }}$ is the power consumption of each multi-rotor UAV in hovering and given as [38]

$$
P_{\text {hov }}=\sqrt{\frac{\left(m_{\mathrm{U}} g\right)^{3}}{2 \pi R^{2} N_{p} \rho}},
$$

where $m_{\mathrm{U}}$ is the mass of UAV in $\mathrm{kg}, g$ is referred as acceleration of gravity in $\mathrm{m} / \mathrm{s}^{2}, R$ and $N_{p}$ denote the propeller radius and number of propellers, respectively, $\rho$ denotes the air density in $\mathrm{kg} / \mathrm{m}^{3}$. In this paper, we adapted the power consumption model for small-cell wireless networks in [39], where $\triangle_{\mathrm{G}}$ is the load-dependent power consumption slope of the ground SBS and $P_{\mathrm{RF}}$ being the RF output power of the ground SBS.

\section{A HYBRID CACHING SCHEME}

In this section, we propose the hybrid caching scheme where UAVs and ground SBSs in small-cell networks cache the contents according to their popularity. In particular, contents are segregated into three groups according to their popularity measure based on the placement probability of $1\left(b_{k}=1\right)$ for the MPC files, between 0 and $1\left(0<b_{k}<1\right)$ for files with the moderate popularity, and the placement probability of $0\left(b_{k}=0\right)$ for the unpopular files that do not need to be cached in the small-cell network. We assume that the content request follows the Zipf distribution where the content request probability is modeled as [40]

$$
m_{k}=\frac{k^{-v}}{\sum_{s=1}^{K} s^{-v}}, \quad v \geq 0,
$$

where $v$ is the Zipf parameter defines the popularity distribution.

In this paper, we seek to improve the overall successful content delivery performance of the hybrid network by the proposed caching scheme. Firstly, we assume that the content placement is made on the basis of MPC scheme which stores the popular content in UAVs and ground SBSs with $b_{k}=1$. In this case, the entire caching capacity is designated to store only the popular content and hence, successful content delivery probability of a hybrid network can be given as

$$
\begin{aligned}
& \mathcal{P}_{\mathrm{SCD}}^{\mathrm{MPC}}(\eta)= \\
& \sum_{k=1}^{J} m_{k} \mathcal{P}_{\mathrm{Cov}}^{\mathrm{U}}\left(\eta, b_{k}=1\right)+\sum_{k=1}^{J} m_{k} \mathcal{P}_{\mathrm{Cov}}^{\mathrm{G}}\left(\eta, b_{k}=1\right),
\end{aligned}
$$

where $\mathcal{P}_{\text {Cov }}^{\mathrm{U}}$ and $\mathcal{P}_{\text {Cov }}^{\mathrm{G}}$ are given in (30) and (37), respectively.

Secondly, we propose the improved caching scheme where a fraction of SBS caching capacity $J_{o}$ is assigned to cache the most popular content called the MPC portion. Thereafter, the contents with the moderate popularity are stored in the remaining portion of the caching capacity called the content diversity (CD) portion. In the CD portion, the disparity of the cached content with less popularity measures represents the content diversity. To this end, the successful content delivery probability of the hybrid network can be given as

$$
\begin{aligned}
& \mathcal{P}_{\mathrm{SCD}}^{\text {Hybrid }}(\eta)= \\
& \sum_{k=1}^{J_{\mathrm{o}}} m_{k} \mathcal{P}_{\mathrm{Cov}}^{\mathrm{U}}\left(\eta, b_{k}=1\right)+\sum_{k=J_{\mathrm{o}}+1}^{J} m_{k} \mathcal{P}_{\mathrm{Cov}}^{\mathrm{U}}\left(\eta, b_{k}^{\prime}\right) \\
& +\sum_{k=1}^{J} m_{k} \mathcal{P}_{\mathrm{Cov}}^{\mathrm{G}}\left(\eta, b_{k}=1\right)+\sum_{k=J_{\mathrm{o}}+1}^{J} m_{k} \mathcal{P}_{\mathrm{Cov}}^{\mathrm{G}}\left(\eta, b_{k}^{\prime}\right) .
\end{aligned}
$$

where $J_{O}$ is defined as the caching capacity in UAVs and ground SBSs to increase the content diversity and hence, improve the overall successful content delivery probability for the hybrid networks. In (46), contents $\left\{1, \cdots, J_{\mathrm{o}}\right\}$ are in the MPC portion of the UAV and ground SBS caches with the placement probability $b_{k}=1$ and contents $\left\{J_{o}+1, \cdots, J\right\}$ are in the $\mathrm{CD}$ portion with the placement probability $0<b_{k}^{\prime}<1$.

\section{NumericAl Results AND Discussion}

In this section, we present and discuss the numerical results. The user association probability in (16) and (23) analyzes the performance of the typical user associated with the UAV and SBS that has the desired cached contents. The successful content delivery probability in (30) and (37) characterizes the downlink transmission performance of the contents cached in 
TABLE II

SYSTEM PARAMETERS

\begin{tabular}{c|l}
\hline Parameters & Values \\
\hline Transmit power of each UAV $\left(P_{\mathrm{U}}\right)$ & $1 \mathrm{~W}$ \\
\hline Transmit power of each ground SBS $\left(P_{\mathrm{G}}\right)$ & $1 \mathrm{~W}$ \\
\hline noise power $\left(\sigma^{2}\right)$ & $-170 \mathrm{dBm}$ \\
\hline frequency $(f)$ and speed of light $(c)$ & $2 \mathrm{GHz}, 3 \times 10^{8} \mathrm{~m} / \mathrm{s}$ \\
\hline Path-loss exponents $\left(\alpha_{\mathrm{L}}, \alpha_{\mathrm{N}}, \alpha_{\mathrm{G}}\right)$ & $2.1,3.7,3.7[11]$ \\
\hline Environment parameter $(a, b)$ & $\begin{array}{l}5.0188,0.3511 \quad \text { Subur- } \\
\text { ban })[34]\end{array}$ \\
\hline Density of hybrid network $\left(\lambda_{\mathrm{S}}\right)$ & $10^{-4} 1 / \mathrm{m}^{2}$ \\
\hline Bit rate of each file $(\varphi / \tau)$ & $100 \mathrm{Kbps}$ \\
\hline Bandwidth $\left(\mathcal{W}_{\mathrm{U}}, \mathcal{W}_{\mathrm{G}}\right)$ & $200 \mathrm{KHz}$ \\
\hline Database size $(K)$ & $10^{4}$ \\
\hline Cache capacity of SBS $(J)$ & $100 \mathrm{files}$ \\
\hline
\end{tabular}

the UAV and SBS. The energy efficiency in (42) evaluates the power consumption performance of a hybrid network while successfully transmitting the most popular contents to the typical user. Furthermore, the successful content delivery performance of the proposed caching scheme in (46) is examined and compared with the popular caching scheme in (45) to characterize the impact of different network parameters.

The simulation and analytical results are obtained by using MATLAB. The simulation results are obtained by using Monte Carlo with $10^{5}$ runs. The system parameters are given in Table II, unless otherwise specified. In Table II, the values for the path-loss exponents and the environment specific parameters are obtained from [11] and [34], respectively, and other values are set for illustration purpose only.

\section{A. User Association Probability}

In Fig. 2, we investigate the impact of the density $\eta$ on the user association probability in suburban environment. In the density sharing scheme, as $\eta$ increases, the density of UAVs increases. As a result, the user association probability with UAVs monotonically increases with $\eta$. Meanwhile, the density of the ground SBSs decreases. Therefore, user association probability monotonically decreases with increase of $\eta$. Thus, we observe that the typical user tends to connect to a tier with higher density.

In Fig. 3, the impact of UAV altitude on the association probability of UAVs is investigated. One can notice that the maximum association probability is 0.98 for $\eta=0.7$ at the altitude of $25.50 \mathrm{~m}$ and is 0.93 for $\eta=0.2$ at the altitude of $46 \mathrm{~m}$. Thus, there exist an optimal UAV altitude to achieve higher association probability which depends on $\eta$ and can be computed by searching (16) numerically. Finally, simulation results are plotted with the markers and agree well with the analytical results of Lemma 1 and Lemma 2 plotted with the solid lines, which validates our analysis.

\section{B. Successful Content Delivery Performance}

In Fig. 4 and Fig. 5, we analyze the effect of the density factor and the UAV altitude, respectively, on the coverage probability. In both cases, the optimal density control factor

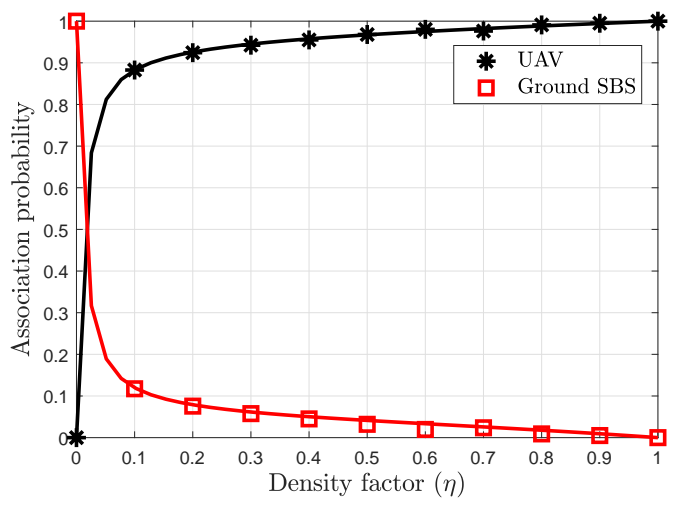

Fig. 2. The impact of SBS density control parameter $(\eta)$ on the user association probability for the UAV and the ground SBS in Suburban environment with $b_{k}=1$.

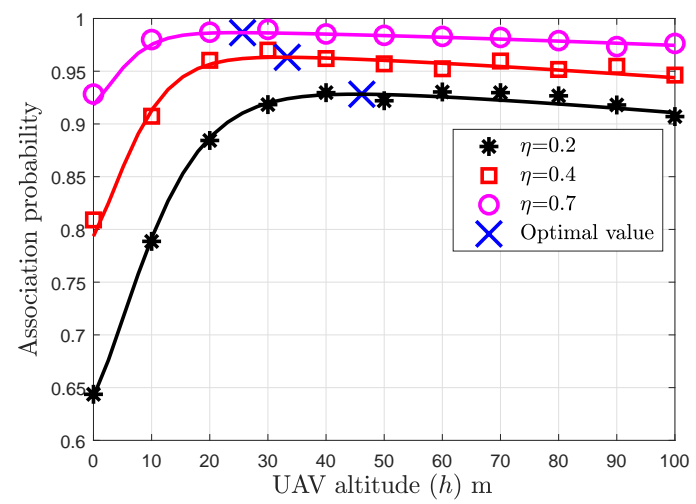

Fig. 3. The impact of UAV altitude $(h)$ on the user association probability for the UAVs with $b_{k}=1$ and different $\eta$.

and the optimal UAV altitude exists for the UAV network. From Fig. 4, it is observed that, an optimal density control parameter exists for an adequate deployment of UAVs, which maximizes the coverage probability. Furthermore, it is observed that the optimal $\eta$ is a function of the given UAV altitude, for instance, $\eta$ is 0.2 and 0.1 for the UAV altitude of 30 and $60 \mathrm{~m}$ to achieve the maximum coverage probability of 0.8 and 0.7 , respectively. On the other hand, the coverage performance of the ground network degrades as $\eta$ increases. However, no significant improvement in the coverage performance is observed for the SBS in the ground network.

For the given density control factor in Fig. 5, the optimal UAV altitude exists for suburban and urban environments. Higher optimal altitude experiences more blockage. On the other hand, the UAVs should fly as low as possible in suburban scenario where there are less blockage. In suburban scenario, as $\eta$ increases from 0.2 to 0.7 , the number of UAVs increases, which increase the amount of co-channel interference generated by cache-enabled UAVs in a network. Therefore, the maximum coverage performance in suburban scenario is achieved at the lower optimal altitudes and at the lesser density factor, for instance, $h$ is 18,22 , and $28 \mathrm{~m}$ for $\eta$ of $0.2,0.4$, and 0.7 to achieve the coverage probability of 0.84 .0 .82 , and 


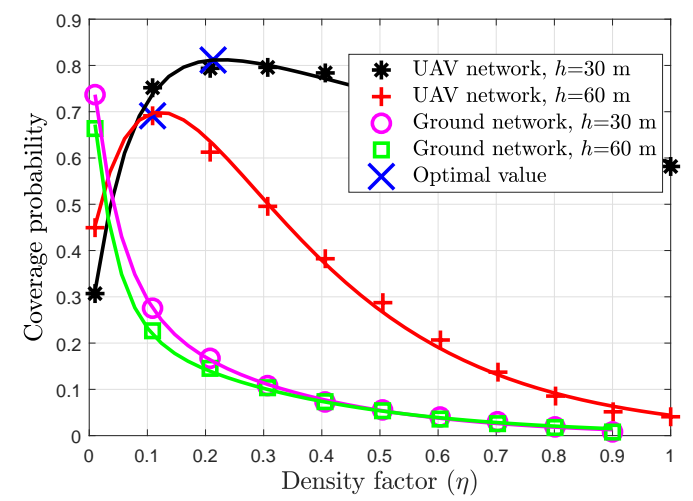

Fig. 4. The impact of the density factor $(\eta)$ on the coverage probability of the UAV and ground network for different altitudes.

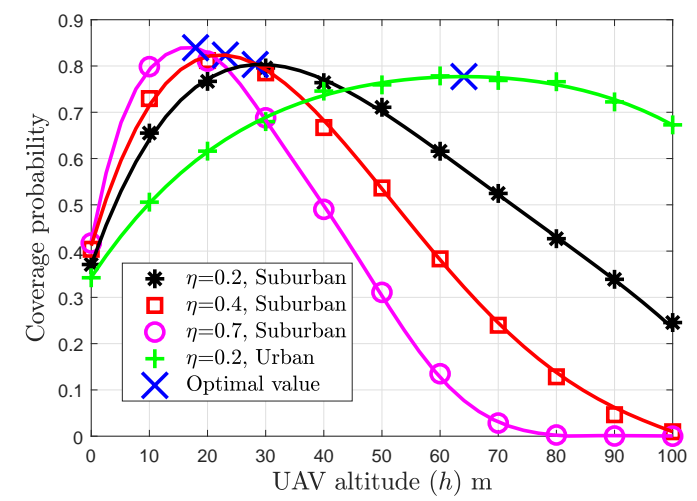

Fig. 5. The impact of the UAV altitude $(h)$ on the coverage probability of the UAV network for different density factor $(\eta)$ in Suburban, and Urban $(a=9.61, b=0.16)$ environments.

0.8 , respectively. Furthermore, the optimal altitude in urban environment is $63 \mathrm{~m}$ for $\eta=0.2$. In Fig. 4 and Fig. 5, the solid line represent the analytical results of Lemma 3 and Lemma 4 while the markers represent the simulation results.

In Fig. 6, we compare the successful content delivery performance for the networks composed solely of UAV, ground SBS and hybrid network with different caching schemes. One can observe that the hybrid network operated with the proposed caching scheme outperform the popular caching scheme implemented for the UAV, ground and hybrid networks. This result is important which shows that the deployment of UAV and ground SBS alone does not meet the increasing content delivery demand in multi-tier heterogeneous network.

\section{Energy Efficiency}

Fig. 7 shows the energy efficiency in the UAV, ground, and hybrid networks as a function of the density control factor with $\delta_{\mathrm{U}}=\delta_{\mathrm{G}}=\delta=0 \mathrm{~dB}$. The parameters concerning the power consumption model of hovering UAV are adapted from [38] with $m_{\mathrm{U}}=0.75 \mathrm{Kg}, g=9.8 \mathrm{~m} / \mathrm{s}^{2}, R=0.2 \mathrm{~m}$ and $N_{p}=4$ (quadcopter UAV), and $\rho=1.225 \mathrm{~kg} / \mathrm{m}^{3}$. Moreover, the parameters for the power consumption model of the ground SBS are used for the femto-cell in [39] with $P_{\mathrm{G}}=4.8 \mathrm{~W}$, $\triangle_{\mathrm{G}}=8.0$, and $P_{\mathrm{RF}}=0.05 \mathrm{~W}$. The energy efficiency of the

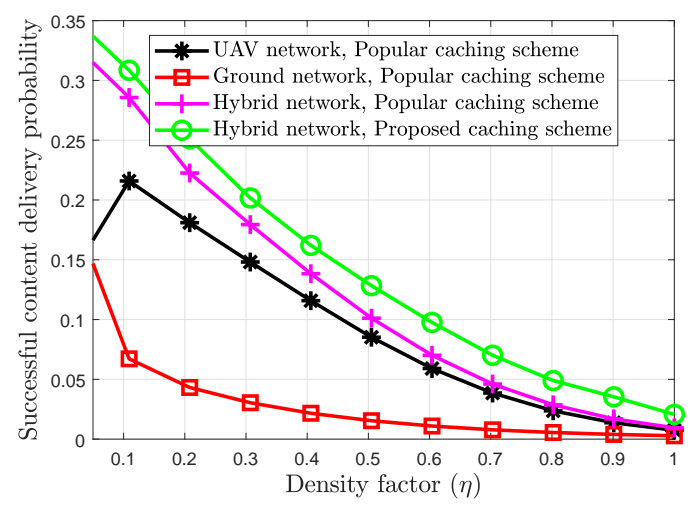

Fig. 6. The impact of the density factor $(\eta)$ on the successful content delivery probability of the UAV, ground and hybrid networks with $J_{o}=50$ files, $h=60 \mathrm{~m}$, and $v=0.8$ for different caching schemes.

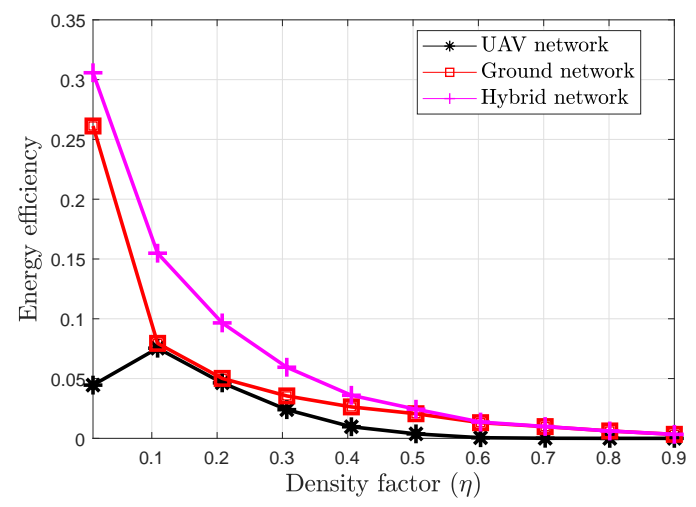

Fig. 7. The impact of the density factor $(\eta)$ on the energy efficiency of the UAV, ground and hybrid networks with $J=100$ files, $h=60 \mathrm{~m}$, and $v=0.8$.

UAV network is worst due to its higher power expenditure to maintain hovering of UAV. From Fig. 7, one can see that the energy efficiency of the ground network is better for femtocell BS when compared to the UAV network. The reason for this is that the RF power of ground SBSs is smaller than the hovering power of UAVs. Finally, it can be seen that energy efficiency of the hybrid network is better than the UAV or ground networks, but beyond $\eta=0.5$, the energy efficiency of the hybrid network is comparable with the ground network.

\section{Performance of the Proposed Caching Scheme}

In Fig. 8 - Fig. 11, we study the effects of caching size, Zipf parameter, UAV altitude, and target data rate of content transmission, respectively, on the successful content delivery performance of the popular caching scheme [22]- [25] and the proposed caching schemes in a hybrid network. The popular caching scheme corresponds to the case when only the most popular files with $b_{k}=1$ are cached in UAVs and ground SBSs. On the other hand, the proposed caching scheme allow to cache two types of files. First, the most popular files. Second, the next most popular files with caching probability $0<b_{k}^{\prime}<1$. 


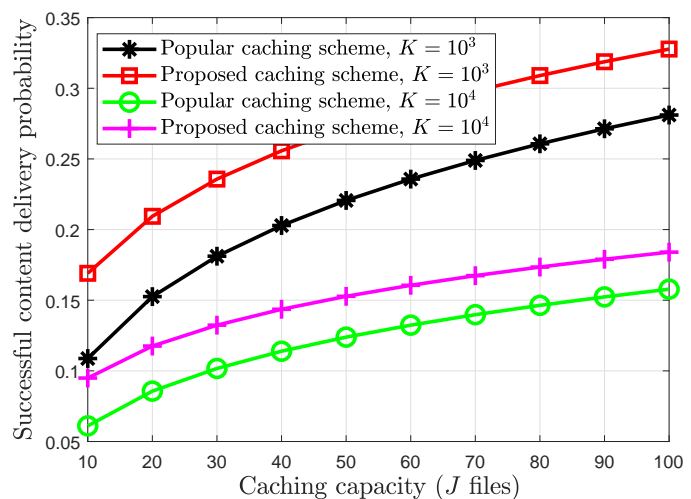

Fig. 8. The impact of the caching capacity on the successful content delivery probability with $J_{O}=50$ files, $\eta=0.7, h=60 \mathrm{~m}$, and $v=0.8$ for different caching schemes.

In Fig. 8, it is observed that the proposed caching scheme performs better with the performance improvement of $26.6 \%$ on average in the successful content delivery over the popular caching scheme which ignores the content diversity. When the content popularity is not uniform it is imperative to consider the content diversity in the probabilistic caching scheme to improve the the content delivery performance. In addition, more different files can be cached at UAVs and SBSs with higher content diversity. In general, as the content size in the database decreases, the probability of the successful content delivery increases with the same performance gap. In Fig. 9 , the successful content delivery performance depends on the skewness of the content popularity distribution defined by $v$. However, for the highly skewed popular content e.g $v>1.6$, the proposed scheme performs as well as popular content placement scheme because very few popular contents are requested by majority of users. In Fig. 10, one can see the existence of the optimal UAV altitude due to the trade-off by the altitude on the successful content delivery performance. When the LOS probability increases, for e.g from 10 to 15 $\mathrm{m}$, the content delivery performance improves due to less shadowing. However, beyond $15 \mathrm{~m}$, the adverse effect on performance occurs due to the greater link distance between UAV and the ground user which corresponds to higher pathloss. In Fig. 11, it is observed that the content delivery probability increases as the target data rate reduces due to the decrease of the SINR threshold and thus, decreases the quality-of-service of the typical user.

\section{CONCLUSION}

In this paper, we have derived the user association probability and the successful content delivery probability and compared the successful content delivery performance of the popular and the proposed caching schemes in the hybrid cacheenabled network. The successful content delivery performance has been improved by $26.6 \%$ on average. Furthermore, the cache-enabled hybrid network is more energy efficient than the separate UAV and ground networks caching the same contents. Future extension of this work includes the study

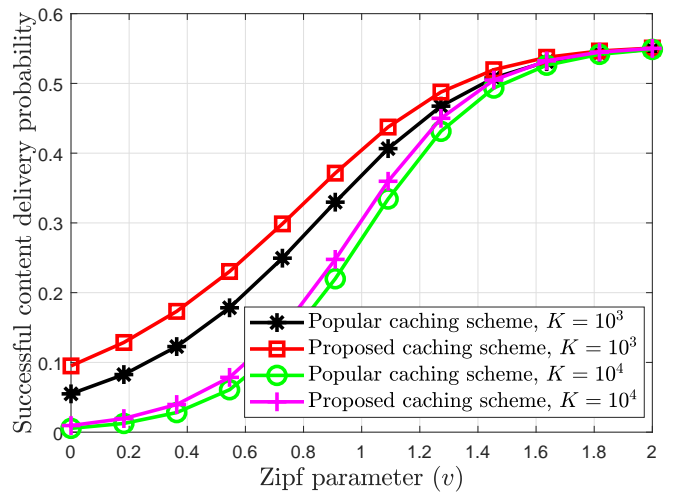

Fig. 9. The impact of popularity skewness of contents on the successful content delivery probability for $\eta=0.7, h=60 \mathrm{~m}$, and $J_{o}=50$ files with different caching schemes.

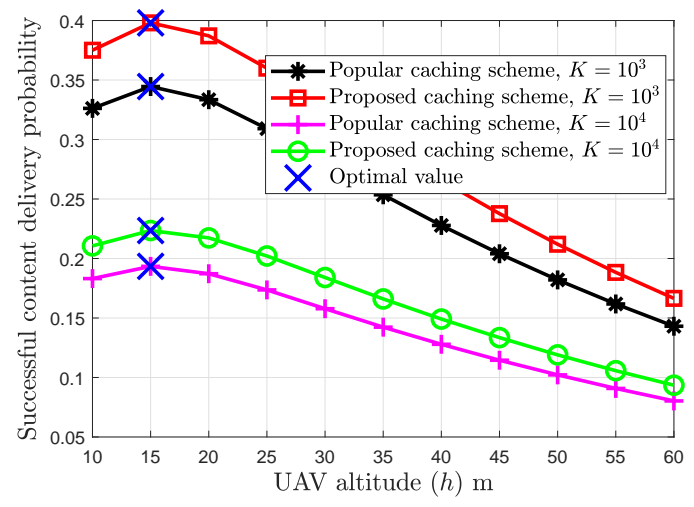

Fig. 10. The impact of the UAV altitude on the successful content delivery probability for $\eta=0.7, h=60 \mathrm{~m}, v=0.8$, and $J_{o}=50$ files with different caching schemes.

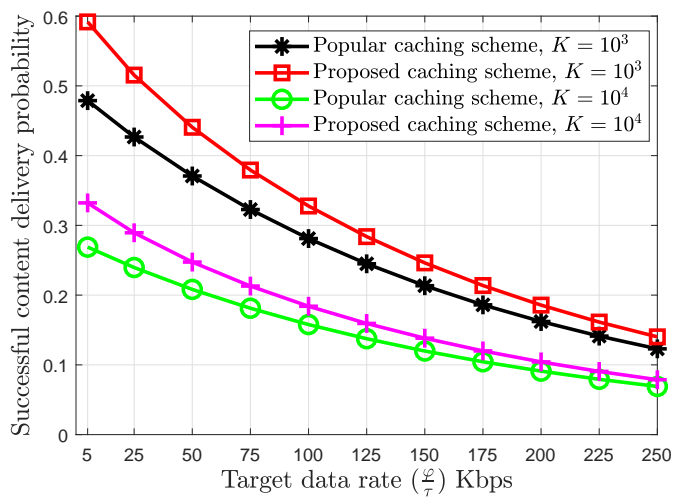

Fig. 11. The impact of target data rate of the link on the successful content delivery probability for $\eta=0.7, h=60 \mathrm{~m}, v=0.8$, and $J_{o}=50$ files with different caching schemes. 
of cooperative caching where UAVs and ground SBSs cache different contents.

\section{REFERENCES}

[1] K. Shanmugam, N. Golrezaei, A. G. Dimakis, A. F. Molisch, and C. Caire, "FemtoCaching: Wireless content delivery through distributed caching helpers," IEEE Trans. Inf. Theory, vol. 59, no. 12, pp. 84028413, Dec. 2013.

[2] N. Golrezaei, A. F. Molisch, A. G. Dimakis, and C. Caire, "Femtocaching and device-to-device collaboration: A new architecture for wireless video distribution," IEEE Commun. Mag., vol. 51, no. 4, pp. 142-149, Apr. 2013.

[3] T. Q. S. Quek, W. C. Cheung, and M. Kountouris, "Energy efficiency analysis of two-tier heterogeneous networks," in IEEE European Wireless Conf., Apr. 2011, pp. 1-5.

[4] Y. Zeng, R. Zhang, and T. J. Lim, "Wireless communications with unmanned aerial vehicles: Opportunities and challenges," IEEE Commun. Mag., vol. 54, no. 5, pp. 36-42, May 2016.

[5] A. A. Khuwaja, Y. Chen, N. Zhao, M.-S. Alouini, and P. Dobbins, "A survey of channel modeling for UAV communications," IEEE Commun. Surv. Tuts., vol. 20, no. 4, pp. 2804-2821, 4th Quart. 2018.

[6] M. Mozaffari, W. Saad, M. Bennis, and M. Debbah, "Efficient deployment of multiple unmanned aerial vehicles for optimal wireless coverage," IEEE Commun. Lett., vol. 20, no. 8, pp. 1647-1650, Aug. 2016.

[7] A. A. Khuwaja, G. Zheng, Y. Chen, and W. Feng, "Optimum deployment of multiple UAVs for coverage area maximization in the presence of cochannel interference," IEEE Access, vol. 7, pp. 85203-85212, 2019.

[8] C. Zhang and W. Zhang, "Spectrum sharing in drone small cells," in Proc. IEEE Global Commun. Conf. (GLOBECOM), Dec. 2016, pp. 1-6.

[9] L. Qi, S. Yan, and M. Peng, "Modeling and performance analysis in UAV assisted ultra dense networks," in Proc. IEEE Int. Conf. Commun. (ICC), Jul. 2018, pp. 1-6.

[10] H. Wu, X. Tao, N. Zhang, and X. Shen, "Cooperative UAV cluster assisted terrestrial cellular networks for ubiquitous coverage," IEEE J. Sel. Areas Commun., vol. 36, no. 9, pp. 2045-2058, Aug. 2018.

[11] M. M. Azari, F. Rosas, and S. Pollin, "Cellular connectivity for UAVs: Network modeling, performance analysis, and design guidelines," IEEE Trans. Wireless Commun., vol.18, no.7, pp. 3366-3381, July 2019.

[12] E. Turgut and M. C. Gursoy, "Downlink analysis in unmanned aerial vehicle (UAV) assisted cellular networks with clustered users," IEEE Access, vol. 6, pp. 36313-36324, 2018.

[13] D. Kim, J. Lee, and T. Q. S. Quek, "Multi-layer unmanned aerial vehicle networks: Modeling and performance analysis," IEEE Trans. Wireless Commun., vol. 19, no. 1, pp. 325-339, Jan. 2020.

[14] X. Lin, J. Xia, and Z. Wang, "Probabilistic caching placement in UAVassisted heterogeneous wireless networks," Phys. Commun., vol. 33, pp. 54-61, Apr. 2019

[15] R. Amer, W. Saad, H. ElSawy, M. M. Butt, and N. Marchetti, "Caching to the sky: Performance analysis of cache-assisted CoMP for cellularconnected UAVs," in IEEE Wireless Commun. Netw. Conf. (WCNC), Apr. 2019, pp. 1-6.

[16] N. Zhao, F. Cheng, F. R. Yu, J. Tang, Y. Chen, G. Gui, and H. Sari, "Caching UAV assisted secure transmission in hyper-dense networks based on interference alignment," IEEE Trans. Commun., vol. 66, no. 5, pp. 2281-2294, May 2018.

[17] N. Zhao, F. R. Yu, L. Fan, Y. Chen, J. Tang, A. Nallanathan, and V. C. M. Leung, "Caching unmanned aerial vehicle-enabled small-cell networks: Employing energy-efficient methods that store and retrieve popular content," IEEE Veh. Technol. Mag., vol. 14, no. 1, pp. 71-79, Mar. 2019.

[18] M. Chen, M. Mozaffari, W. Saad, C. Yin, M. Debbah, and C. S. Hong, "Caching in the sky: Proactive deployment of cache-enabled unmanned aerial vehicles for optimized quality-of-experience," IEEE J. Sel. Areas Commun., vol. 35, no. 5, pp. 1046-1061, May 2017.

[19] M. G. Khoshkholgh, K. Navaie, H. Yanikomeroglu, V. C. M. Leung, and K. G. Shin, "Randomized caching in cooperative UAV-enabled fogRAN," in IEEE Wireless Commun. Netw. Conf. (WCNC), Apr. 2019, pp. $1-6$.

[20] F. Zhou, N. Wang, G. Luo, L. Fan, and W. Chen, "Edge caching in multi-UAV-enabled radio access networks: 3D modeling and spectral efficiency optimization," IEEE Trans. Signal Inf. Process. Over Netw., vol. 6, pp. 329-341, Apr. 2020.
[21] J. Wen, K. Huang, S. Yang, and V. O. Li, "Cache-enabled heterogeneous cellular networks: Optimal tier-level content placement," IEEE Trans. Wireless Commun., vol. 16, no. 9, pp. 5939-5952, Sept. 2017.

[22] Z. Chen, J. Lee, T. Q. Quek, and M, Kountouris, "Cooperative caching and transmission design in cluster centric small cell networks," IEEE Trans. Wireless Commun., vol. 16, no. 5, pp. 3401-3415, May 2017.

[23] G. Zheng, H. A. Suraweera, and I. Krikidis, "Optimization of hybrid cache placement for collaborative relaying," IEEE Commun. Lett., vol. 21, no. 2, pp. 442-445, Feb. 2017.

[24] C. Zhan and G. Yao, "SVC video delivery in cache-enabled wireless HetNet," IEEE Syst. J., vol. 12, no. 4, pp. 3885-3888, Dec. 2018.

[25] Y. Zhu, G. Zheng, L. Wang, K. Wong, and L. Zhao, "Content placement in cache-enabled sub- $6 \mathrm{GHz}$ and millimeter-wave multi-antenna dense small cell networks," IEEE Trans. Wireless Commun., vol. 17, no. 5, pp. 2843-2856, May 2018.

[26] R. Wang, R. Li, E. Liu, and P. Wang, "Performance analysis and optimization of caching placement in heterogeneous wireless networks," IEEE Commun. Lett., vol. 23, no. 10, pp. 1883-1887, Oct. 2019.

[27] L. Enamipour, Z. Zeinalpour-Yazdi, and B. H. Khalaj, "Performance analysis of cache-enabled wireless networks considering stochastic geometry approach," IET Communications, vol. 13, no. 8, pp. 1043-1050, May 2019.

[28] S. Zhang, W. Sun, and J. Liu, "An optimized spatially cooperative caching strategy for heterogeneous caching network," in $15^{\text {th }}$ Int. Wireless Commun. \& Mobile Comput. Conf. (IWCMC), Jun. 2019, pp. 1685-1689.

[29] T. Zhang, X. Xu, L. Zhou, X. Jiang, and J. Loo, "Cache space efficient caching scheme for content-centric mobile Ad hoc networks," IEEE Syst. J., vol. 13, no. 1, pp. 530-541, Mar. 2019.

[30] M. Haenggi, Stochastic geometry for wireless networks. Cambridge University Press, 2012.

[31] M. Haenggi, J. G. Andrews, F. Baccelli, O. Dousse, and M. Franceschetti, "Stochastic geometry and random graphs for the analysis and design of wireless networks," IEEE J. Sel. Areas Commun., vol. 27, no. 7, pp. 1029-1046, Sept. 2009.

[32] Y. S. Soh, T. Q. S. Quek, M. Kountouris, and H. Shin, "Energy efficient heterogeneous cellular networks," IEEE J. Sel. Areas Commun., vol. 31, no. 5, pp. 840-850, May 2013.

[33] A. Al-Hourani, S. Kandeepan, and A. Jamalipour, "Modeling air-toground path loss for low altitude platforms in urban environments," Proc. IEEE Global Commun. Conf. (GLOBECOM), Dec. 2014, pp. 2898-2904.

[34] A. Al-Hourani, S. Kandeepan, and S. Lardner, "Optimal LAP altitude for maximum coverage," IEEE Wireless Commun. Lett., vol. 3, no. 6 , pp. 569-572, Dec. 2014.

[35] H. Chae, and W. Choi, "Caching placement in stochastic wireless caching helper networks: Channel selection diversity via caching," IEEE Trans. Wireless Commun., vol. 15, no. 10, pp. 6626-6637, Oct. 2016.

[36] Y. Cui and D. Jiang, "Analysis and optimization of caching and multicasting in large-scale cache-enabled heterogeneous wireless networks," IEEE Trans. Wireless Commun., vol. 16, no. 1, pp. 250-264, Jan. 2017.

[37] Y. Cui, D. Jiang, and Y. Wu, "Analysis and optimization of caching and multicasting in large-scale cache-enabled wireless networks," IEEE Trans. Wireless Commun., vol. 15, no. 7, pp. 5101-5112, Jul. 2016.

[38] H. Ghazzai, M. Ben Ghorbel, A. Kadri, M. J. Hossain, and H. Menouar, "Energy-efficient management of unmanned aerial vehicles for underlay cognitive radio systems," IEEE Trans. Green Commun. Netw., vol. 1, no. 4, pp. 434-443, Dec. 2017.

[39] G. Auer, V. Giannini, C. Desset, I. Godor, P. Skillermark, M. Olsson, M. A. Imran, D. Sabella, M. J. Gonzalez, O. Blume, and A. Fehske, "How much energy is needed to run a wireless network?" IEEE Wireless Commun. Mag., vol. 18, no. 5, pp. 40-49, Oct. 2011.

[40] L. Breslau, P. Cao, L. Fan, G. Phillips, and S. Shenker, "Web caching and zipf-like distributions: evidence and implications," in Proc. IEEE Conf. on Computer Commun. (INFOCOM), Mar. 1999, pp. 126-134. 


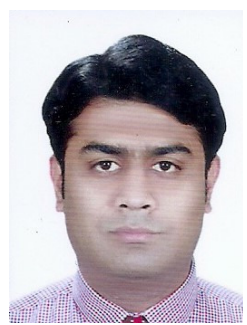

Aziz Altaf Khuwaja received his B.E. degree in telecommunication engineering from Mehran University of Engineering \& Technology, Jamshoro, Pakistan, in 2010 and M.Sc. in electronics communication and computer engineering from the University of Nottingham, U.K. in 2015. He is currently pursuing $\mathrm{Ph} . \mathrm{D}$. in Engineering at the School of Engineering, the University of Warwick, U.K. He is also working as an Assistant Professor at the Department of Electrical Engineering, Sukkur IBA University, Sukkur, Pakistan. His research interests include wireless communications and radio resource management in UAV communications.

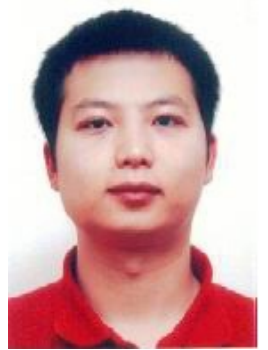

Wei Liu (M'08-SM'14) obtained the B.S. degree from the University of Electronic Science and Technology of China, Chengdu, China, in 1999, the M.S. degree from Xidian University, Xi'an, China, in 2003, respectively, and the Ph.D. degree from the University of Southampton, U.K. Since 2007, he has been with Xidian University, China. His current research interests include MIMO communications systems, and interference management, and joint communication, computation and caching.

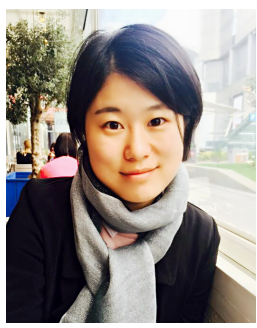

Yongxu Zhu (S'16-M'19) received the Ph.D degree in Electrical Engineering from University College London in 2017. From 2017 to 2019, she was a Research Associate with Loughborough University. She is currently a Lecturer (Assistant Professor) with the Division of Computer Science and informatics, London South Bank University, since 2019. She has served as an Editor of the IEEE Wireless Communication Letters. Her research interests include large intelligent surface Communications, UAV communications, wireless edge caching, millimeter-wave communications, heterogeneous cellular networks, physical-layer security and block-chain.

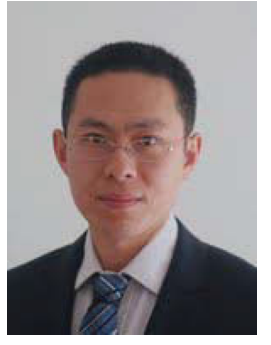

Gan Zheng (S'05-M'09-SM'12) received the BEng and the MEng from Tianjin University, Tianjin, China, in 2002 and 2004, respectively, both in Electronic and Information Engineering, and the $\mathrm{PhD}$ degree in Electrical and Electronic Engineering from The University of Hong Kong in 2008. He is currently Reader of Signal Processing for Wireless Communications in the Wolfson School of Mechanical, Electrical and Manufacturing Engineering, Loughborough University, UK. His research interests include machine learning for communications, wireless power transfer, UAV communications, mobile edge caching and fullduplex radio. He is the first recipient for the 2013 IEEE Signal Processing Letters Best Paper Award, and he also received 2015 GLOBECOM Best Paper Award, and 2018 IEEE Technical Committee on Green Communications Computing Best Paper Award. He was listed as a Highly Cited Researcher by Thomson Reuters/Clarivate Analytics in 2019. He currently serves as an Associate Editor for IEEE Communications Letters and IEEE Wireless Communications Letters.

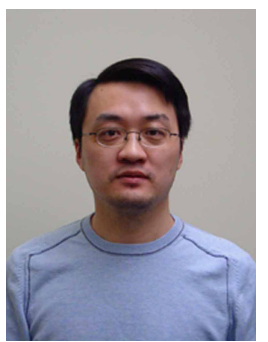

Yunfei Chen (S'02-M'06-SM'10) received his B.E. and M.E. degrees in electronics engineering from Shanghai Jiaotong University, Shanghai, P.R.China, in 1998 and 2001, respectively. He received his Ph.D. degree from the University of Alberta in 2006. $\mathrm{He}$ is currently working as an Associate Professor at the University of Warwick, U.K. His research interests include wireless communications, cognitive radios, wireless relaying and energy harvesting. 